\title{
Maladies membranaires de l'intestin et du rein
}

Les membranes cellulaires participent au maintien d'un niveau stable de métabolites indispensables au bon fonctionnement de l'organisme. Ce rôle primordial est illustré par les exemples connus de maladies membranaires qui affectent les voies normales d'assimilation des glucides et des protéines dans l'intestin et le rein.

\section{Alfred Berteloot, Christiane Malo}

Groupe de recherche en transport membranaire, Université de Montréal, Québec.

\section{REFERENCES}

I. Crane RK. Enzymes and malabsorption: A concept of brush border membrane disease. Gastroenterology 1966; 50: 254-62.

2. Gray GM. Carbohydrate absorption and malabsorption. In: Johnson LA, ed. Physiology of the Gastrointestinal Tract. New York: Raven Press, 1981: 1097-122.

3. Crane RK. Digestion and absorption: Watersoluble organics. In: Crane RK, ed. Internat Rev Physiol. Baltimore: Univ Park Press, 1977: 32565.

4. Silk DBA, Dawson AM. Intestinal absorption of carbohydrate and protein in man. In: Crane RK, ed. Internat Rev Physiol. Baltimore: Univ. Park Press, 1979: 151-204.

5. Crane RK. The gradient hypothesis and other models of carrier-mediated active transport. Rev Physiol Biochem Pharmacol 1977; 78: 99-159.

\section{ADRESSE}

A. Berteloot, Ch. Malo : Groupe de recherche en transport membranaire, Département de physiologie, Faculté de médecine, Université de Montréal, CP 6208, Succursale A, Montréal, Québec, $\mathrm{H}_{3} \mathrm{C}{ }_{3} \mathrm{~T} 8$. a notion de maladie membranaire, introduite par Crane [I] dès I966, caractérise une fonction membranaire fortement diminuée ou inexistante du fait de la réduction ou de l'absence totale d'activité normalement associée à une protéine spécifique des membranes cellulaires. Les tissus épithéliaux de l'intestin grêle et du segment proximal du tubule rénal, dont les fonctions sont essentielles au maintien de l'homéostasie métabolique de l'organisme, ont été particulièrement bien étudiés à cet égard. Il faut cependant distinguer entre affections primaires et secondaires. Les premières, qui retiendront notre attention, représentent des modifications spécifiques, sous contrôle génétique, dans la structure ou la régulation d'une protéine membranaire particulière; les secondes, consécutives à une variété de conditions pathologiques, produisent des modifications membranaires généralisées souvent partielles et réversibles.

\section{Les fonctions \\ membranaires}

Les concentrations plasmatiques d'acides aminés et de monosaccharides sont le résultat d'un équilibre dynamique entre digestion et absorption intestinales, métabolisme cellulaire et réabsorption rénale. Les protéines et les polysaccharides d'origine alimentaire sont tout d'abord scindés en molécules plus petites par les enzymes digestives d'origine pancréatique qui agissent dans la lumière intestinale [2]. Les étapes finales de la digestion incombent aux hydrolases présentes dans la membrane de bordures en brosse (MBB) des entérocytes (tableau I et figure, voie $I$ : voir p. 428-9) $[3,4]$. L'absorption des produits finaux d'hydrolyse (figure, voies 2-4) est assurée par des protéines spécifiques également présentes dans ces membranes (tableau II : voir p. 43I) [3, 4]. Le transport des molécules vers l'intérieur des cellules s'effectue suivant leurs propres gradients de concentration par un processus de diffusion facilitée (figure, voies 2 et 3 ) ou contre un gradient de concentration par transport actif (figure, voie 4). L'énergie requise pour soutenir ce dernier est fournie par le gradient électrochimique de $\mathrm{Na}^{+}$dont le maintien est assuré par la $\mathrm{Na}^{+}$$\mathrm{K}^{+}$-ATPase présente dans toutes les membranes baso-latérales (MBL) des cellules épithéliales (figure) [5]. Elle est ensuite récupérée grâce à un couplage entre le substrat et le $\mathrm{Na}^{+}$au niveau d'un transporteur membranaire (figure, voie 4 et tableau II) [5]. Les substrats absorbés ont alors accès au milieu sanguin, après passage à travers la MBL des cellules, en général par diffusion facilitée (figure, voie 5) [5] et sont ainsi véhiculés vers les différents organes. En même temps, les acides aminés libres et les monosaccharides produits par le métabolisme de ces différents organes sont également déversés dans la circulation sanguine. Quant au segment 


\section{REFERENCES}

6. Ullrich KJ. Sugar, amino acid, and $\mathrm{Na}^{+}$ cotransport in the proximal tubule. Ann Rev Physiol 1979; 41: 181-95.

7. Murer H, Ahearn G, Biber J, Cassano G, Gmaj P, Stieger B. Co- and counter-transport mechanisms in brush border membranes and basal-lateral membranes of intestine and kidney. 7 Exp Biol 1983; 106: 163-80.

8. Matthews DM. Intestinal absorption of peptides. Physiol Rev 1975; 55: 537-608.

9. Schmitz J, Triadou N. Digestion et absorption intestinales des peptides. Gastroenterol Clin Biol $1982 ; 6: 651-61$.

10. Brot-Laroche E Alvarado F. Disaccharide uptake by brush-border membrane vesicles lacking the corresponding hydrolases. Biochim Biophys Acta 1984; 775: 175-81.

11. Turner RJ, Moran A. Heterogeneity of sodium-dependent D-glucose transport sites along the proximal tubule: evidence from vesicle studies. Am J Physiol 1982 ; 242: F406-14.

12. Dorando FC, Crane RK. Studies on the kinetics of $\mathrm{Na}^{+}$gradient-coupled glucose transport as found in brush-border membrane vesicles from rabbit jejunum. Biochim Biophys Acta 1984; 772: 273-87.

13. Stevens BR, Kaunitz JD, Wright EM. Intestinal transport of amino acids and sugars: advances using membrane vesicles. Ann Rev Physiol $1984 ; 46: 417-33$

14. Berteloot A. Characteristics of glutamic acid transport by rabbit intestinal brush-border membrane vesicles. Effects of $\mathrm{Na}^{+}-, \mathrm{K}^{+}$- and $\mathrm{H}^{+}$- gradients. Biochim Biophys Acta 1984; 775: 129-40.

15. Sacktor B. L-glutamate transport in rena plasma membrane vesicles. Mol Cell Biochem $1981 ; 39: 239-51$.

16. Christensen HN. Organic ion transport during seven decades. The amino acids. Biochim Biophys Acta 1984; 779: 255-69.

17. Ramaswamy K, Malathi P, Crane RK. Demonstration of hydrolase-related glucose transport in brush border membrane vesicles prepared from guinea pig small intestine. Biochem Biophys Res Commun 1976; 68: 162-8.

18. Berteloot A, Khan AH, Ramaswamy K. Characteristics of dipeptide transport in normal and papain-treated brush border membrane vesicles from mouse intestine. I. Uptake of glycyl-Lphenylalanine. Biochim Biophys Acta 1981; 649: 179-88.

19. Berteloot A, Khan AH, Ramaswamy K. Characteristics of dipeptide transport in normal and papain-treated brush border membrane vesicles from mouse intestine. 2. Uptake of glycyl-Lleucine. Biochim Biophys Acta 1982; 686: 47-54.

20. Rajendran VM, Berteloot A, Ishikawa $\mathrm{Y}$, Khan AH, Ramaswamy K. Transport of carnosine by mouse intestinal brush-border membrane

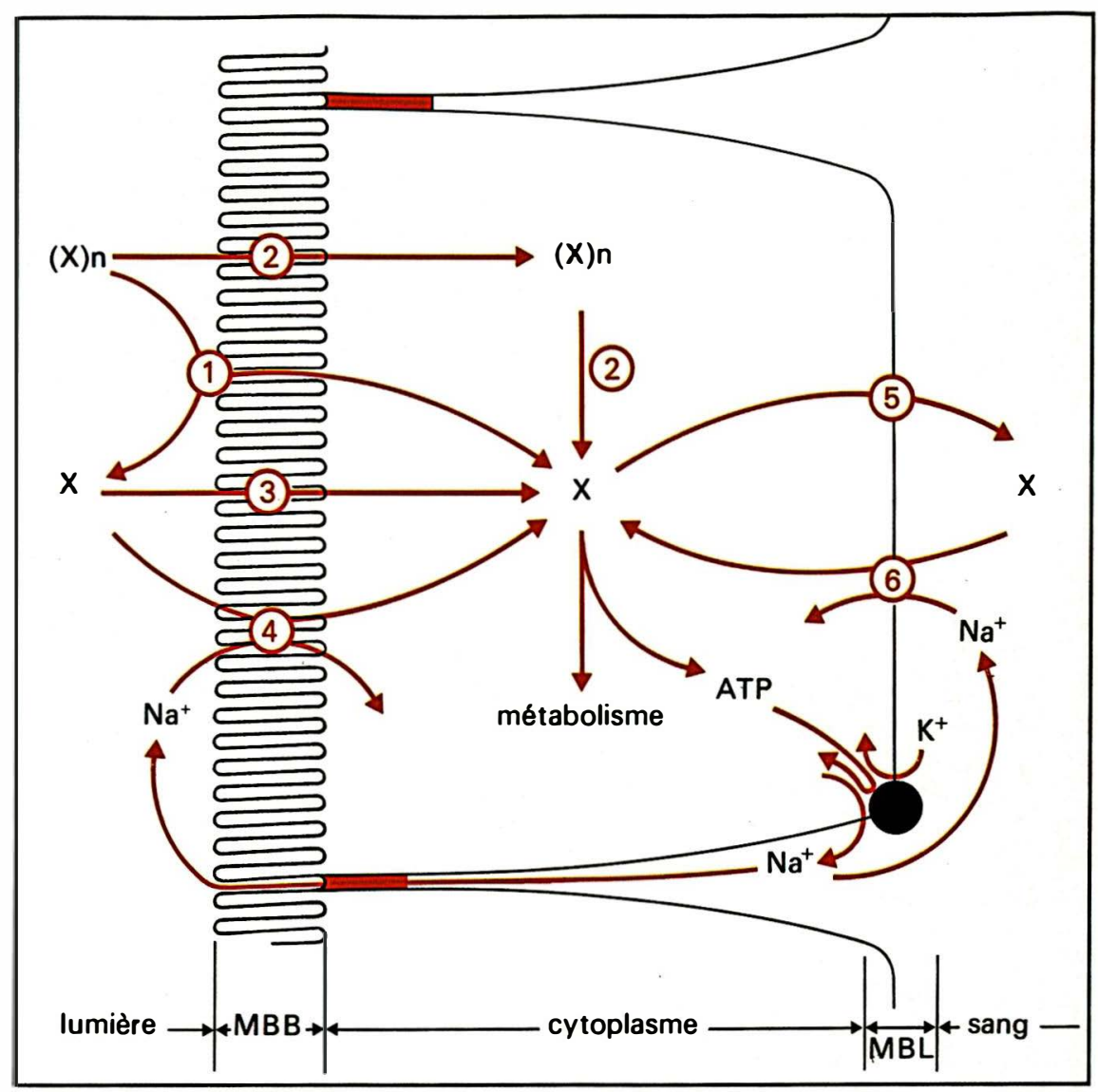

Figure. Voies membranaires d'assimilation des sucres et des protéines $\left(\mathrm{X}_{\boldsymbol{n}}\right)$ au niveau des cellules épithéliales de l'intestin et du rein.

I. Hydrolyse finale des disaccharides $(n=2)$ et des peptides $(n<8)$ par les enzymes de la $M B B$ (tableau I) conduisant à la formation de monosaccharides et d'acides aminés $(X)$ libres. Une action vectorielle de ces enzymes, qui permettrait un accès privilégié des sucres et acides aminés constitutifs des disaccharides et des peptides vers l'intérieur des cellules, a été suggérée par les études in vivo mais semble réfutée par les expériences sur vésicules membranaires (voie pointillée).

2. Voie d'absorption des peptides $(2 \leqslant n<4)$ intacts suivie de l'hydrolyse intracellulaire en acides aminés par les peptidases cytoplasmiques. Une multiplicité de transporteurs membranaires est possible mais reste à préciser. Une voie de ce type n'existe pas pour les disaccharides.

3. Voie de transport apical des sucres et des acides aminés par diffusion facilitée.

4. Voie de transport apical des sucres et des acides aminés énergisée par couplage au gradient électrochimique de $\mathrm{Na}^{+}$produit par le fonctionnement de la $\mathrm{Na}^{+}, \mathrm{K}^{+}-$ ATPase des $M B L$.

5. Voie de transport basolatéral des sucres et des acides aminés par diffusion facilitée. 6. Voie de transport basolatéral des acides aminés couplée au gradient électrochimique de $\mathrm{Na}^{+}$. Elle servirait à fournir la cellule en acides aminés indispensables à son métabolisme. Toutefois, le système ASC, doué de capacités d'échange importantes, pourrait également être utilisé lors du transport transcellulaire des acides aminés. L'existence de systèmes de ce type pour les sucres est possible mais non démontrée sans ambiguité.

Le tableau II (page 43I) détaille les différents systèmes de transport connus et fonctionnant selon les voies 2 à 6 . 
proximal du rein, il assure, dans les conditions normales, une réabsorption quasi totale de ces molécules après filtration glomérulaire. Là également, la réabsorption doit se faire par passage consécutif à travers la MBB (figure, voies $I-4$ ) et la MBL (figure, voie 5) des cellules épithéliales du tubule rénal et met en jeu des composantes protéiques membranaires spécifiques (tableau II) $[6,7]$. Plusieurs enzymes sont également présentes au niveau des MBB du segment proximal du tubule rénal (tableau 1 ) [3], mais leur rôle y est difficile à déterminer étant donné les faibles concentrations du filtrat glomérulaire en substrats qu'elles hydrolysent.

Toute modification dans les processus responsables du maintien des concentrations plasmatiques en métabolites essentiels, induite par une déficience intestinale et/ou rénale ou consécutive à une perturbation métabolique, va donc entraîner des variations du taux de ces métabolites dans le sang et/ou les urines et se traduira parfois par un état pathologique. De plus, la polarisation des cellules épithéliales du rein et de l'intestin et la présence de systèmes de transport actifs et passifs au sein des MBB et des MBL font ressortir la possibilité d'une absorption (figure, voies $I-4+5$ ) ou d'une sécrétion (figure, voies $6+3)$ nettes selon les concen- trations luminales, intracellulaires et sanguines des différents métabolites. Dans les conditions normales, le bilan est en faveur d'une absorption et les systèmes $\mathrm{MBL}$ actifs servent essentiellement à pourvoir les cellules épithéliales en substrats indispensables à leur métabolisme. Toutefois, une déficience en transport actif MBB pourrait inverser ce bilan.

\section{Relations entre hydrolyse et transport}

Les voies générales d'absorption illustrées dans la figure sont utilisées à des degrés divers, selon la complexité (nombre de liaisons osidiques ou peptidiques) et la nature (glucidique ou protéique) des molécules présentes dans les lumières intestinale ou tubulaire. Le tableau II présente en détails les systèmes membranaires spécifiques de l'intestin et du rein qui participent à l'assimilation des différentes catégories de molécules [2-4, 8-I6].

Il existe encore un grand nombre de spéculations quant aux interrelations spatiales et fonctionnelles entre l'hydrolyse membranaire des disaccharides et des peptides et les processus de transport intestinal des monosaccharides et des acides aminés résultant de cette digestion [2-4, $8,9]$. Il est en effet difficile de concilier le fait que l'hydrolyse n'est pas limitante pour l'absorption in vivo avec l'existence d'un avantage cinétique pour le transport que présentent les produits d'hydrolyse par rapport aux monosaccharides et acides aminés luminaux. De plus, la démonstration in vitro selon laquelle les disaccharides peuvent agir comme enzymes vectorielles en transférant les produits de réaction directement à travers la membrane (figure, voie $I$ : pointillés) ne prouve pas l'importance physiologique de ce processus connu sous le terme générique de HRT (Hydrolase-Related Transport) [17]. Toutefois, l'existence de transporteurs spécifiques pour les disaccharides et la possibilité d'un fonctionnement vectoriel des peptidases (selon le mode du HRT) dans le transport des peptides sont déniés par les études sur vésicules de $\mathrm{MBB}$ intestinales $[10,18-20]$.

Il apparaît donc que les disaccharides et les oligopeptides (plus de 3 acides aminés) doivent être hydrolysés avant de pouvoir être absorbés (figure, voie I) [8, 10]. Pour les di- et tripeptides, l'importance relative de l'hydrolyse et de l'absorption (figure, voies I et 2) semble essentiellement fixée par la susceptibilité des peptides aux hydrolases membranaires [18-20]. L'existence de transporteur(s) capable(s) d'assurer le passage de di- et tripeptides intacts à travers la $\mathrm{MBB}$

\begin{tabular}{|c|c|c|c|}
\hline \multicolumn{4}{|c|}{$\begin{array}{c}\text { Tableau I } \\
\text { ENZYMES DE LA MBB IMPLIQUÉES DANS L'HYDROLYSE DES GLUCIDES ET DES PEPTIDES }\end{array}$} \\
\hline \multirow{2}{*}{ enzymes } & \multirow{2}{*}{ spécificité } & \multicolumn{2}{|c|}{ localisation tissulaire } \\
\hline & & intestin & rein \\
\hline $\begin{array}{l}\text { saccharidases } \\
\text { saccharase-isomaltase } \\
\text { lactase-phlorizine-hydrolase } \\
\text { tréhalase } \\
\text { maltase } \\
\text { glucoamylase }\end{array}$ & $\begin{array}{l}\text { saccharose et } \alpha \text {-dextrines ramifiées } \\
\text { lactose et glycosylcéramides } \\
\text { tréhalose } \\
\text { maltose } \\
\text { amidon ( } \alpha \text {-dextrines ramifiées) }\end{array}$ & $\begin{array}{l}+ \\
+ \\
+ \\
+ \\
+\end{array}$ & $\begin{array}{l}- \\
+ \\
+ \\
-\end{array}$ \\
\hline $\begin{array}{l}\text { peptidases } \\
\text { dipeptidyl-aminopeptidase IV } \\
\text { aminopeptidase } \mathrm{N} \\
\text { aminopeptidase A } \\
\text { oligopeptidases } \\
\text { entéropeptidase } \\
\gamma \text {-glutamyltransférase }\end{array}$ & $\begin{array}{l}\text { hydrolyse de peptides à proline antépénultième } \\
\text { hydrolyse d'acides aminés neutres et basiques } \\
N \text {-terminaux } \\
\text { hydrolyse d'acides aminés acides } N \text {-terminaux } \\
\text { oligopeptides } \\
\text { activation du trypsinogène en trypsine } \\
\text { transfert de groupement } \gamma \text {-glutamyl du glutathion } \\
\text { sur un acide aminé ou un peptide }\end{array}$ & $\begin{array}{l}+ \\
+ \\
+ \\
+ \\
+ \\
+\end{array}$ & $\begin{array}{l}+ \\
+ \\
+ \\
+ \\
+\end{array}$ \\
\hline
\end{tabular}


(figure, voie 2) et distinct(s) de ceux utilisés par les acides aminés libres a été amplement vérifiée $[8,9]$ et il apparaît que le mécanisme mis en jeu est indépendant de la présence d'un gradient de $\mathrm{Na}^{+}$[18-20].

\section{Maladies membranaires}

En théorie, la liste des maladies membranaires de l'intestin et du rein pourrait être aussi longue que celle des molécules individuelles, de nature protéique ou lipidique, connues ou inconnues, qui constituent les MBB et MBL des deux types de cellules épithéliales. En pratique, on ne connaît à présent qu'un nombre limité de maladies qui affectent essentiellement quelques-uns des systèmes enzymatiques (tableau III) ou de transport (tableau IV) connus au niveau de ces membranes $[2-4,21]$.

L'analyse de la figure permet de prévoir les modifications susceptibles d'induire des maladies par perturbation des fonctions normales de digestion et de transport aux niveaux rénal et intestinal ainsi que de classifier les différents types de maladies membranaires selon les cibles qui auront été atteintes. Ainsi, l'absence d'une activité hydrolytique dans les MBB intestinales pourra provoquer une malabsorption spécifique pour certains disaccharides (qui requièrent obligatoirement une disaccharidase pour l'hydrolyse ou un transport via le HRT) mais n'aura que peu d'effets sur la digestion des protéines (puisque celle-ci pourra être assurée par les protéases pancréatiques). De même, une telle absence dans les MBB rénales sera sans conséquence étant donné les faibles concentrations du filtrat glomérulaire en substrats pour ces enzymes. C'est ce que démontre clairement le tableau III. En effet, toute déficience de la MBB intestinale en disaccharidase se traduit par une malabsorption après ingestion du substrat spécifique, facilement corrigée par le retrait du disaccharide du régime alimentaire $[4,22]$. Pour la MBB rénale, par contre, aucune déficience en hydrolase n'a été rapportée, et ce, probablement, en raison de l'absence de manifestation clinique associée à une telle anomalie.
L'analyse de la figure permet également de prévoir qu'une déficience en un système de transport luminal (MBB) pourra réduire l'absorption intestinale ou la réabsorption rénale d'un substrat ou d'un groupe de substrats. C'est effectivement ce qui est observé (tableau IV), sauf pour les peptides et les acides $\beta$-aminés. Dans ces deux cas, il est probable que la multiplicité des transporteurs pour les peptides et/ou l'hydrolyse possible de ces substrats avant transport d'une part, et que la capacité qu'ont les acides $\beta$-aminés d'utiliser le transporteur des acides aminés neutres aux niveaux rénal [13] et intestinal d'autre part [14], font que de telles déficiences seraient asymptomatiques et pourraient passer inaperçues pour cette raison même. Il est également intéressant de constater dans le tableau $I V$ que la plupart des syndromes de malabsorption pour les acides aminés sont asymptomatiques (sauf pour la présence d'acides aminés particuliers dans les selles et les urines), ce qui s'explique très bien par la multiplicité des systèmes de transport et leurs spécificités croisées pour différents groupes de molécules. Cependant, si ces déficiences s'accompagnent d'une carence nutritionnelle, l'homéostasie métabolique est perturbée et des troubles se manifestent [21]. D'autre part, on peut encore remarquer que les acides aminés impliqués dans les malabsorptions intestinales diminuent dans les selles de patients atteints, quand la forme libre de ces molécules est supprimée de leur alimentation. Cette constatation souligne l'importance de la voie d'absorption des peptides du point de vue nutritionnel $[4,8]$. La situation est toutefois differente pour les autres. Le tableau IV fait ressortir que les rares cas de malabsorption congénitale du glucose et du galactose, caractérisés par une déficience spécifique des systèmes intestinal et rénal responsables du transport actif de ces deux monosaccharides à travers les MBB, sont associés à des troubles cliniques graves. L'absence de rôle physiologique de HRT, indiquée par cette observation, semble confirmée par l'absorption négligeable de glucose constatée après perf usion enté- 


\begin{tabular}{|c|c|c|c|c|c|c|}
\hline \multicolumn{7}{|c|}{$\begin{array}{l}\text { Tableau II } \\
\text { ASSIMILATION DES GLUCIDES ET DES PROTIDES }\left(X_{n}\right)\end{array}$} \\
\hline \multirow{2}{*}{\multicolumn{2}{|c|}{$X_{n}$}} & \multicolumn{2}{|c|}{$\begin{array}{l}\text { Voies d'assimilation } \\
\text { selon figure }\end{array}$} & \multirow[t]{2}{*}{$\begin{array}{l}\text { Substrats } \\
\text { types }\end{array}$} & \multirow[t]{2}{*}{ Commentaires } & \multirow[t]{2}{*}{ Ref. } \\
\hline & & MBB & MBL & & & \\
\hline \multirow{2}{*}{$\begin{array}{l}+ \\
\Delta N \\
\end{array}$} & dextrines & $\begin{array}{l}1 \text { (glucoamylase } \\
\text { et isomaltase) }\end{array}$ & - & voir & $\begin{array}{l}\text { Hydrolyse obligatoire } \\
\text { avant transport. Cellules } \\
\text { intestinales uniquement }\end{array}$ & 2,3 \\
\hline & oligopeptides & 1 (oligopeptidase $(\mathrm{s})$ ) & - & . & $\begin{array}{l}\text { hydrolyse obligatoire avant } \\
\text { transport }\end{array}$ & 4,8 \\
\hline \multirow{2}{*}{$\begin{array}{l}v \\
v \\
c \\
V / \\
v\end{array}$} & $\begin{array}{l}\text { tripeptides } \\
\text { et dipeptides }\end{array}$ & $\begin{array}{l}1 \text { (peptidases) } \\
2 \text { (transporteur(s)) }\end{array}$ & $\bar{?}$ & \multirow{2}{*}{$\begin{array}{l}\text { voir } \\
\text { tableau I }\end{array}$} & \begin{tabular}{|l|} 
relations entre hydrolyse et \\
transport imprécises
\end{tabular} & \multirow{2}{*}{$\begin{array}{c}4,8 \\
9 \\
2,10\end{array}$} \\
\hline & disaccharides & 1 (disaccharidases) & - & & $\begin{array}{l}\text { hydrolyse obligatoire avant } \\
\text { transport }\end{array}$ & \\
\hline & fructose & 3 (transporteur) & $?$ & fructose & $\begin{array}{l}\text { métabolisme cellulaire } \\
\text { important }\end{array}$ & 3,4 \\
\hline & $\begin{array}{l}\text { glucose } \\
\text { et } \\
\text { galactose }\end{array}$ & 4 (transporteur(s)) & $\begin{array}{l}5 \text { (transporteur) } \\
6 \text { (transporteur)? }\end{array}$ & $\begin{array}{c}\text { glucose } \\
\text { et } \\
\text { galactose }\end{array}$ & $\begin{array}{l}2 \text { transporteurs au niveau } \\
\text { des MBB rénales mais } \\
\text { situation imprécise pour } \\
\text { l'intestin. Système MBL à } \\
\text { préciser }\end{array}$ & $\begin{array}{c}3,11 \\
12\end{array}$ \\
\hline \multirow{7}{*}{ 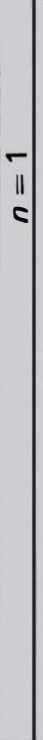 } & \multirow{7}{*}{$\begin{array}{l}\text { acides } \\
\text { aminés } \\
\text { neutres }\end{array}$} & 4 (NBB) & - & & $\begin{array}{l}\text { exclusif aux MBB rénales } \\
\text { et instestinales }\end{array}$ & \multirow{7}{*}{13} \\
\hline & & 4 (imino) & - & $\begin{array}{l}\text { acides } \\
\text { iminés, pro }\end{array}$ & très sélectif & \\
\hline & & 4 (PHE) & - & phe et met & & \\
\hline & & $4(\beta)$ & - & $\begin{array}{l}\beta \text {-ala, } \\
\text { taurine }\end{array}$ & $\begin{array}{lll}\text { cellules rénales uni- } & \\
\text { quement } & \end{array}$ & \\
\hline & & $3(L)$ & $5(L)$ & $\begin{array}{c}\text { chaînes } \\
\text { hydrophobes }\end{array}$ & peu sélectif & \\
\hline & & - & $6(A)$ & $\begin{array}{l}\text { chaînes } \\
\text { courtes } \\
\text { (Ala) }\end{array}$ & $\begin{array}{l}\text { Peu sélectif - Sujet à régu- } \\
\text { lation }\end{array}$ & \\
\hline & & - & 6 (ASC) & $\begin{array}{c}\text { chaînes } \\
\text { à } 3 \text { et } 4 \\
\text { carbones } \\
\text { (Ala, Ser, Cys) }\end{array}$ & $\begin{array}{l}\text { très stéréospécifique - } \\
\text { Capacité d'échange }\end{array}$ & \\
\hline & $\begin{array}{l}\text { acides aminés } \\
\text { basiques }\end{array}$ & $3\left(Y^{+}\right)$ & $5\left(y^{+}\right)$ & Lys, Arg & $\begin{array}{l}\text { dépendance sodique } \\
\text { rénale possible }\end{array}$ & \\
\hline & $\begin{array}{l}\text { acides aminés } \\
\text { acides }\end{array}$ & $4\left(X_{A G}\right)$ & $6\left(X_{A G}\right)$ & $\begin{array}{c}\text { L-glu et } \\
L \text { - et D-asp }\end{array}$ & $\begin{array}{l}\text { active par } \mathrm{K}^{+} \text {intracellu- } \\
\text { laire et sensible aux } \mathrm{H}^{+} \text {. } \\
\text { Existence non prouvée } \\
\text { pour MBL intestinales }\end{array}$ & $\begin{array}{l}14 \\
15\end{array}$ \\
\hline
\end{tabular}

La terminologie utilisée pour les transporteurs d'acides aminés est tirée des réfërences [13] ct [16]. 


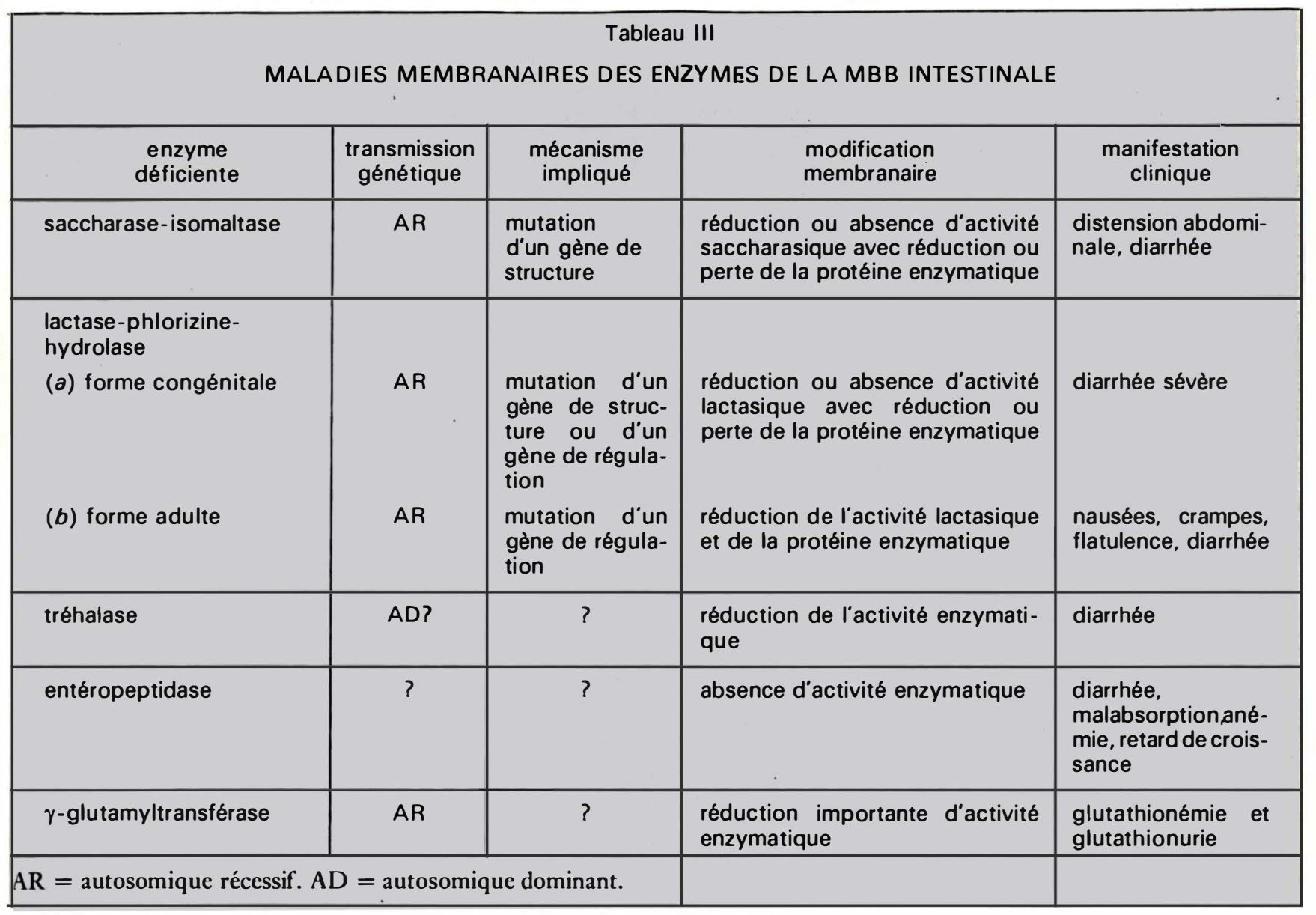

rale de saccharose et de maltose chez deux patients souffrant de cette déficience [4]. On peut donc probablement expliquer l'absence d'effet clinique grave de la glycosurie rénale par le fait qu'elle est spécifique au tubule rénal et pourrait n'affecter que le transporteur de la pars recta $[11,21]$.

La figure permet aussi d'envisager une troisième possibilité qui impliquerait la déficience d'un système de transport au niveau des membranes basolatérales dont la conséquence serait une malabsorption grave pour un groupe de substances (sucres ou acides aminés) et dramatiques quelque soit le tissu affecté, rénal ou intestinal (puisque la voie transcellulaire serait bloquée). Une telle éventualité est en fait démontrée dans le cas de l'intolérance aux protéines et à la lysine (aminoacidurie dibasique de type 2 ). C'est l'absence de transporteur pour les acides aminés basiques des MBL intestinales et rénales (et des membranes plasmiques des hépatocytes) qui cause cette maladie [21]. Finalement, un dernier regard à la figure permet de prédire que les modifications qui affecteraient la capacité d'accumulation intracellulaire des systèmes de cotransport produiraient une malabsorption généralisée de tous les substrats activement transportés. Parmi celles-ci, l'atténuation de l'amplitude du gradient électrochimique de $\mathrm{Na}^{+}$(par réduction de l'activité $\mathrm{Na}^{+}-\mathrm{K}^{+}$-ATPase par exemple) ou l'augmentation de la perméabilité passive des membranes, hypothèse proposée par Bergeron [21], serait susceptible de provoquer ce genre d'anomalie. Il apparaît effectivement que plusieurs maladies héréditaires (syndromes de Fanconi) présentent une malabsorption généralisée pour les sucres et les acides aminés (et d'autres substances éventuellement). Plusieurs ont été reliées à des intoxications métaboliques consécutives à l'accumulation intra- cellulaire d'un intermédiaire métabolique (galactosémie, intolérance au fructose et cystinose, par exemple) mais un certain nombre attendent encore une explication (syndromes de Lowe, de Busby...).

\section{Développement et fonction membranaire}

L'origine génétique des maladies membranaires est un fait clairement établi mais il reste à préciser si la pathogénie implique une mutation dans un gène de structure ou de régulation, voire une pertubation des mécanismes d'expression de ces gènes. L'étude des mécanismes qui contrôlent l'apparition d'une fonction membranaire donnée à un moment précis du développement permet quelques indications.

Les membranes cellulaires du rein et de l'intestin acquièrent en effet leurs caractéristiques fonctionnelles adultes au cours d'un long processus de différenciation, d'un stade 
embryonnaire à une étape de maturité fonctionnelle. Le mode de régulation des fonctions associées à la membrane apicale des cellules rénales demeure à déterminer; en revanche, plusieurs études portant sur le développement des enzymes de la MBB des entérocytes chez les rats et souris nouveau-nés ont clairement démontré l'existence d'un contrôle de type multi-hormonal à ce niveau. Les glucocorticoides et la thyroxine [23], de même que l'insuline [24] et le facteur de croissance épidermique [25], sont autant de substances dont l'influence sur la prolifération et/ou la différenciation des entérocytes a été démontrée. Chaque hormone exerce son effet propre sur l'un ou l'autre aspect du processus de maturation, mais les hormones peuvent aussi agir de concert pour moduler l'expression d'un phénotype particulier [26].

Il semble de plus en plus pertinent de parler de l'effet des hormones en terme de modulation plutôt que de contrôle. Le tissu intestinal apparaît effectivement "programmé " pour franchir, au moment voulu, toutes les étapes de différenciation devant mener au stade adulte. Les hormones n'affecteraient que la chronologie des événements et l'intensité des modifications engendrées. Ceci explique probablement pourquoi les tentatives visant à induire une activité saccharasique chez des individus déficients, par traitement hormonal sont restées sans effet [27], et appuie l'hypothèse qu'une mutation génétique est à l'origine des déficiences membranaires.

L'hypothèse précédente semble confirmée par les études d'incidence familiale des maladies membranaires de l'intestin et du rein. De plus, une analyse globale des tableaux III et IV fait ressortir les éléments suivants :

I. une déficience fonctionnelle de la MBB de l'intestin n'implique pas nécessairement l'existence d'une déficience similaire au niveau du rein et vice-versa. Les entités protéiques responsables des diverses fonctions ne sont donc pas nécessairement identiques dans les membranes des deux types cellulaires et pourraient être codées par des gènes différents. En ce qui concerne les fonctions de transport, seule une purification des transpor-

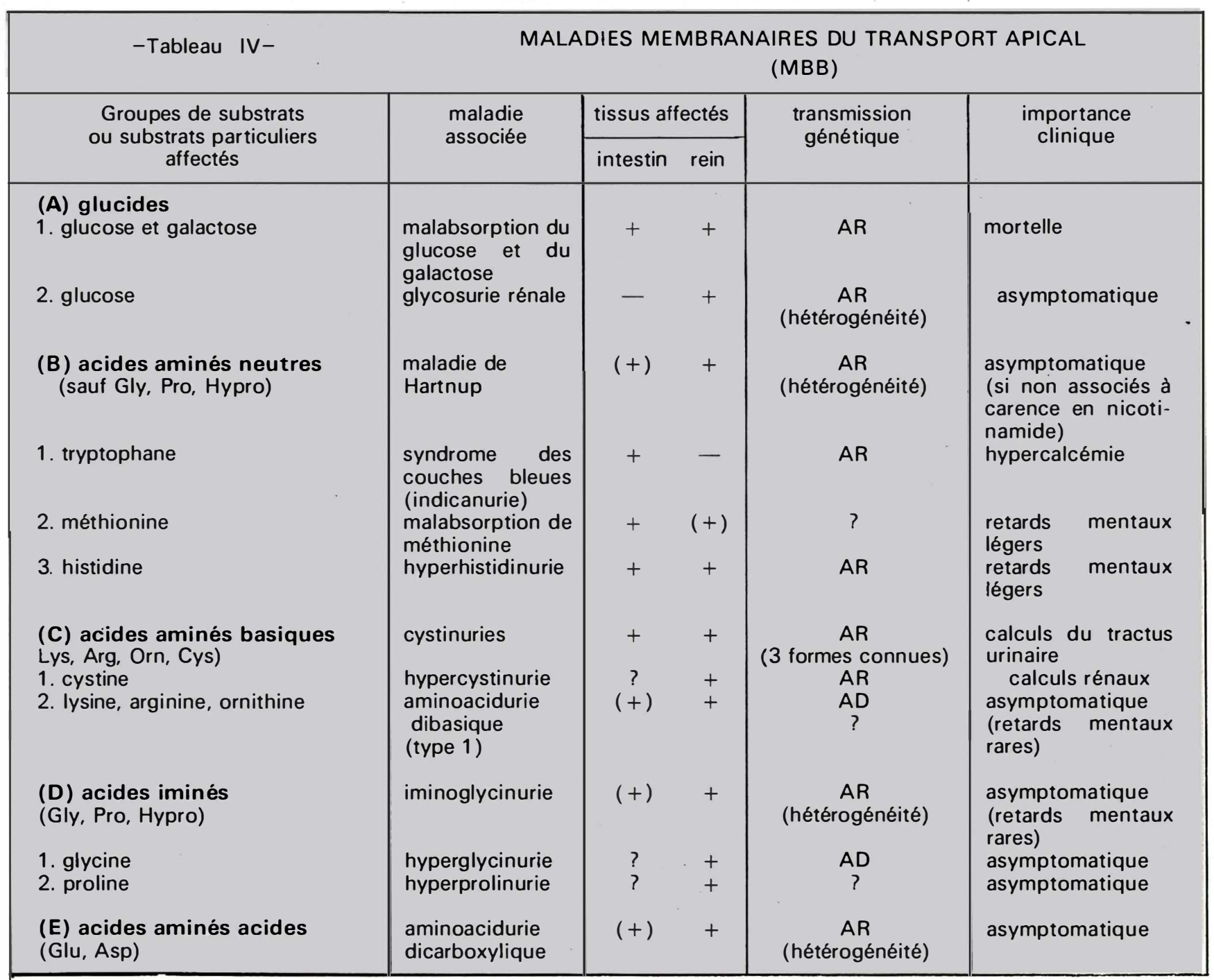

$(+)$ déficience membranaire dont la localisation tissulaire n'est pas nécessairement associée à une même diffërence dans l'autre organe.

$m / s n^{\circ} 8$ décembre 85 
teurs pourrait trancher la question mais elle reste à réaliser. En ce qui concerne les activités hydrolytiques de la MBB, aucune déficience commune aux deux membranes n'a été rapportée chez un même individu; 2. un défaut de transport au niveau de la MBB ne correspond pas nécessairement à une perturbation similaire au niveau des MBL de la même cellule. Cette constatation implique que les protéines fonctionnelles représentent des produits de gènes différents ou des produits de transcription distincts contrôlés à différents loci sur un même gène. L'ignorance actuelle des mécanismes de triage des protéines synthétisées vers leurs membranes respectives (reconnaissance d'un signal sur des protéines similaires ou différentes) ne permet pas de trancher entre ces deux possibilités;

3. les déficiences membranaires sont transmises selon les lois mendéliennes mais le(s) gène(s) impliqué(s) dans la mutation demeure(nt) inconnu(s). En effet, la biogenèse des membranes implique tout un appareil synthétique complexe (glycosyltransférases du réticulum endoplasmique et de l'appareil de Golgi, fusions membranaires, enzymes responsables de la synthèse des lipides, etc.) dont les éléments, tout comme les composantes protéiques membranaires elles-mêmes, sont codés par des gènes de structure dont l'expression pourrait être modulée par des gènes de régulation. Ainsi, toute mutation génétique sur l'un de ces gènes pourrait provoquer la perte ou une modification sélective des protéines responsables des fonctions d'hydrolyse et/ou de transport, ou de la composition en lipides qui modulent physiquement l'expression membranaire de ces fonctions.

\section{Mécanismes moléculaires}

Le développement de techniques d'isolement de membranes, l'analyse des composantes protéiques des MBB par électrophorèse sur gel de polyacrylamide et l'utilisation d'anticorps spécifiques dirigés contre une portion déterminée de la protéine fonctionnelle sont autant d'approches permettant aux biolo- gistes moléculaires d'acquérir une meilleure compréhension des mécanismes impliqués. Jusqu'à présent la plupart des études concernent les enzymes de la MBB intestinale mais on peut penser que les mêmes mécanismes seraient mis en jeu de façon générale dans les déficiences membranaires des deux tissus.

Ces études permettent de conclure qu'une réduction ou une perte d'activité dans la MBB est toujours associée à une disparition partielle ou totale de la bande protéique correspondante après électrophorèse sur gel de polyacrylamide [28].

Ceci a été clairement démontré pour les déficiences en complexes saccharase-isomaltase [29] et lactase-phlorizine hydrolase [30] chez des sujets atteints. De tels résultats sont toutefois compatibles avec un défaut de synthèse, une dégradation accrue d'une forme protéique instable, un défaut d'insertion de la protéine dans la membrane ou encore une perturbation dans les mécanismes de régulation permettant l'expression d'un gène de structure. La mise en évidence de déficiences membranaires a contribué à la caractérisation des diverses voies de transport et à l'identification des fonctions spécifiques d'une composante membranaire donnée. Les maladies membranaires représentent à présent un modèle intéressant pour l'étude de la biogenèse des membranes et de sa régulation. Une analyse comparée des $\mathrm{MBB}$ rénale et intestinale devrait en outre permettre de préciser les analogies fonctionnelles et génétiques existant entre ces deux organes.

D'un point de vue clinique autant que fondamental, il s'avère d'autre part extrêmement important d'approfondir nos connaissances sur la régulation hormonale et sur les mécanismes moléculaires impliqués dans les déficiences congénitales ou acquises. Tant que nous ne saurons pas si la mutation génétique affecte la synthèse d'une ou de plusieurs sous-unités fonctionnelles, l'assemblage ou la formation du complexe actif, la fixation ou l'insertion de ce complexe dans la membrane, ou la vitesse de dégradation de cette protéine fonctionnelle, il sera vain d'espérer intervenir de façon directe pour corriger la situation

\section{Summary}

In normal conditions, plasma levels of metabolites result from intestinal assimilation of food nutrients, organ metabolism, and renal reabsorption from the glomerular filtrate. Epithelial cell membranes from the small intestine and the renal proximal tubule thus play an essential role in the overall process of metabolic homeostasis. Primary membrane diseases in these tissues, characterized as inborn errors of membrane functions, are discussed in terms of normal transcellular sugar and amino acids assimilation and classified according to potential sites of defective pathways. Possible molecular mechanisms involved in the pathogenesis of these diseases are then inferred from typical features of intestinal brush border membrane enzyme deficiencies and regulation and from the comparison between transport def ects associated with intestinal and renal tissues as well as with brush border or basolateral membranes. However, we have yet to learn about the identity of membrane proteins, their synthesis and insertion into different membrane domains, and their genetic regulation before identifying the true nature of the abnormality responsible for the inherited membrane disorders.

\section{TIRES A PART}

A. Berteloot : Groupe de recherche en transport membranaire, Département de physiologie, Faculté de médecine, Université de Montréal, CP 6208, Succursale A, Montréal, Québec, $\mathrm{H}_{3} \mathrm{C}_{3} \mathrm{~T} 8$ 